\title{
Subcellular Localization and Quantification of Camptothecin in Different Plant Parts of Chonemorpha Fragrans
}

\author{
Pradnya Kedari, Nutan Malpathak* \\ Department of Botany, University of Pune, Pune 411007, Maharashtra, India \\ *Corresponding Author: mpathak@unipune.ac.in
}

Copyright (C) 2013 Horizon Research Publishing All rights reserved.

\begin{abstract}
Chonemorpha fragrans (C. fragrans), a liana belonging to family Apocynaceae, shows presence of widely used anticancer compound Camptothecin (CPT). CPT is a modified monoterpene indole alkaloid produced by very few species belonging to unrelated orders of angiosperms, especially plants belonging to the families Apocynaceae and Icacenaceae. In this work a sensitive high performance thin layer chromatography method (HPTLC) was used for detection and quantification of CPT. HPTLC analysis showed that highest amount of CPT was present in root methanolic extract ( $0.023 \%$ of dry weight) followed by stem bark methanolic extract $(0.013 \%$ of dry weight). Also, the subcellular localization of an anticancer drug has been studied using autofluroscence property of CPT under Ultra violet (UV) light. Localization study revealed that out of all the organs studied roots accumulated highest levels of CPT followed by stem bark. Xylem tissues showed a major accumulation site for CPT. Presence of CPT in in vitro culture is an important finding as in vitro systems can be used for further experiments to reduce overexploitation of natural population. This is the first report rearding subcellular localization and quantification of CPT in sequential extracts of different plant parts in C. fragrans.
\end{abstract}

Keywords Chonemorpha Fragrans, Apocynaceae, Anticancer, Camptothecin, HPLC, HPTLC, Localization

\section{Introduction}

C. fragrans (Moon) Alston. (Apocynaceae) is an unexplored medicinal plant belonging to Western Ghats. Scanty information has been available so far on the composition and activity of secondary metabolites in $C$. fragrans. Roots of this plant have wide importance in folk medicines. Recently an anticancer compound Camptothecin (CPT) has been reported from C. fragrans [1].
Anti-cancer activity of CPT is due to its ability to inhibit the eukaryotic DNA topoisomerase I [2]. Naturally occurring CPT is important source of chemotherapeutic agents for clinical treatment of cancer. It also inhibits retroviruses such as the human immunodeficiency virus (HIV) [3]. Chemical synthesis of camptothecin is reported but extraction from plant sources is cost effective method. This fact makes the plants more valuable as a source of CPT as there are limited sources available for CPT extraction. The literature survey reveals that the presence of any valuable product from plant becomes the reason for its overexploitation and so for its threatened status. It is essential to find out new sources as well as attempts have to be made in order to boost the CPT production in the available sources. The present study illustrates first time the presence of CPT in the in vitro cultures of $C$. fragrans established in our lab. Localization and quantification of anti-cancer alkaloid Camptothecin (CPT) in vivo and in vitro in the sequential plant extracts of $C$. fragrans has been investigated in this communication. These results have important implications for harnessing the high yielding individuals for clonal multiplication. Presence of such drug in the cultures will help to prevent the overexploitation as well as could prove an alternative source.

Extracts of root, stem bark, leaves, in vitro shoots and callus in methanol, ethyl acetate, chloroform and hexane were comparatively analyzed for CPT content using high performance thin layer chromatography (HPTLC) and high performance liquid chromatography techniques (HPLC). The quantity of CPT in the extract was estimated on the basis of linear calibration curves obtained using the concentration range of 5 to $50 \mathrm{ng}$ with standard CPT. The subcellular localization of CPT, has been investigated in this work using autoflurescence of CPT in UV light which reveals the cellular site of accumulation of CPT in C. fragrans.

To our knowledge this is the first report of localization of CPT in C. fragrans, serial extraction of CPT and showing presence of CPT in the in vitro shoots and callus in $C$. fragrans. 


\section{Materials and Methods}

\subsection{Localization}

Histochemical analysis was carried out using fresh vegetative organs. Fresh sections of meristem, leaf, petiole, internode, root and callus were analyzed under UV-fluorescent microscope (Olympus research microscope 10X.) for the presence of autofluorescence that CPT molecule emits when exposed to UV radiation.

\subsection{Plant Material}

Plant samples were collected from Kerala $\left(10^{\circ}-46^{\prime} \mathrm{N}\right.$ latitudes and $\left.75^{\circ}-55^{\prime} \mathrm{E}\right)$. The plants were grown in the glasshouse through cuttings and maintained at the Botanic Garden, Department of Botany, University of Pune (Maharashtra, India). Leaf, stem, and root samples were collected from $C$. fragrans trees. In vitro shoots used for experiment were taken from the cultures maintained on B5 medium supplemented with $8.8 \mu \mathrm{m}$ BAP, were air dried and used to prepare the extracts. The callus used was obtained on B5 medium supplemented with $8.8 \mu \mathrm{m}$ BAP $+3.2 \mu \mathrm{m}$ NAA.

\subsection{Extract Preparation}

The plant parts of $C$. fragrans (i.e. leaves, stem bark, roots, in vitro shoots and callus) were collected and shade-dried. This shade dried material was crushed in to fine powder using grinder. $20 \mathrm{~g}$ sample was extracted repeatedly in 150 $\mathrm{ml}$ of hexane and methanol separately for $18 \mathrm{hrs}$ and then the hexane extract residue was sequentially extracted in chloroform and ethyl acetate (Merck, India) using same procedure. The methanolic extract was called as crude extract. All the extracts were evaporated to dryness and redissolved in HPLC-grade methanol. These extracts were filtered using a $0.45 \mu \mathrm{m}$ cellulose nitrate membrane (Laxbro) and stored at $-20^{\circ} \mathrm{C}$ till further analysis was carried out.

\subsection{Analysis}

\subsubsection{Thin-Layer Chromatography (TLC)}

Silica gel coated plates (Merck) were used for the TLC analysis. As standard solution, authentic CPT (Sigma) in DMSO: methanol (1:50) at a concentration of $0.01 \mathrm{mg} / \mathrm{ml}$ was used. The extracts of the plant samples and the standard solution were spotted on a TLC plate $(10 \mathrm{~cm}$ in height and 20 $\mathrm{cm}$ in width) and developed with chloroform: ethyl acetate $(1: 1 \mathrm{v} / \mathrm{v})$ in a saturated chromatography chamber for about $20 \mathrm{~min}$. The plates were visualized in UV light. The TLC analyses revealed that CPT was present.

\subsubsection{High Performance Thin-Layer Chromatography (HPTLC)}

Chromatographic analyses of the extracts were performed on TLC silica gel 60 F254 plates $(10 \times 20 \mathrm{~cm}$; Merck,
Darmstadt, Germany). Aliquots $(10 \mu \mathrm{l})$ of each extract were applied on the plates as bands using an Linomat V Automatic Sample applicator (Camag, Muttenz, Switzerland), Each 5 $\mathrm{mm}$-wide band was separated from its neighboring bands by a distance of $11.4 \mathrm{~mm}$. Plates were developed in a glass twin trough chamber $(20 \times 10 \times 4 \mathrm{~cm})$ (Camag) previously saturated $(20 \mathrm{~min})$ using ethyl acetate:chloroform $(1: 1 \mathrm{v} / \mathrm{v})$ as the mobile phase. The development length was $80 \mathrm{~mm}$ (development time approximately $10 \mathrm{~min}$ ). After development, plates were dried for $10 \mathrm{~min}$. Plates containing methanol extracts and standard CPT (Sigma) were scanned using Camag TLC scanner operated by win CATS software (Camag) under the following conditions: scanning mode, re-emission fluorescence (mercury lamp); measurement wavelength- $366 \mathrm{~nm}$; slit dimension- $4.00 \times 0.03 \mathrm{~mm}$.

\subsubsection{Calibration curve of Camptothecin}

$10 \mu \mathrm{l}$ of each concentration of the standard solutions of CPT was applied on TLC plate. The plate was developed in solvent system chloroform: ethyl acetate $(1: 1 \mathrm{v} / \mathrm{v})$. After development, the plate was dried in air and scanned at 366 $\mathrm{nm}$. Data was used to obtain CPT calibration curve.

\subsubsection{HPLC Analysis}

HPLC analysis of the methanolic extracts of $C$. fragrans was carried out as described in our earlier report [4] with slight modification. Isocratic analytical HPLC was performed with DIONEX (Germany) using RPC18 column, P680 HPLC pump, a $2 \mathrm{ml}$ injection loop (Rheodhyne USA) and UVD 170 detection controlled with Chromeleon software (DIONEX softtron GmbH, Germany). The mobile phase for alkaloid elution was acetonitrile: water (40:60), at a flow rate $1.0 \mathrm{ml} / \mathrm{min}$ with a sample size of $20 \mu \mathrm{l}$ and UV detection at $254 \mathrm{~nm}$. A standard curve was obtained using authentic sample of camptothecin (Sigma Aldrich). The standard was prepared using DMSO: methanol $(1: 50 \mathrm{v} / \mathrm{v})$. Co-chromatography of the extracts was performed with authentic samples for confirmation. Validation of quantitative method was performed for samples in 3 replications. All reagents were HPLC grade.

\subsubsection{Statistical Analysis}

Analysis of variance (one-way ANOVA) was performed to test the significance of differences between means obtained among the treatments in each experiment at the 5\% level of significance $(\mathrm{P}<0.05)$.

\section{Results}

The objective of the present study was to reveal the accumulation of the alkaloid camptothecin (CPT) in both in vitro as well as in vivo plant material of $C$. fragrans. CPT localized in the sections was characterized by a blue fluorescence similar to that shown by the authentic CPT (Sigma) (Figures 1-5). The accumulation of CPT was seen in various tissues of almost all the organs observed. 


\subsection{Roots}

The difference between sections of in vivo and in vitro materials can be seen prominently in the root sections (Figure 1A-F). The in vivo root section showed prominent fluorescence in trichomes, epidermis as well as 3-4 layered hypodermis (Figure 1A,C,E). The in vitro sections showed fluorescence in epidermis but trichomes were absent (Figure $1 \mathrm{~B}, \mathrm{D}, \mathrm{F})$. The endodermis also shows the presence of CPT in in vivo as well as in vitro sections. In mature section the root hair showed presence of CPT and also number of layers showing the fluorescence was more in epidermis. The xylem elements showed prominent fluorescence in both in vivo as well as in vitro sections. Root tip also shows the presence of $\mathrm{CPT}$ in both the sections (Figure 1A).

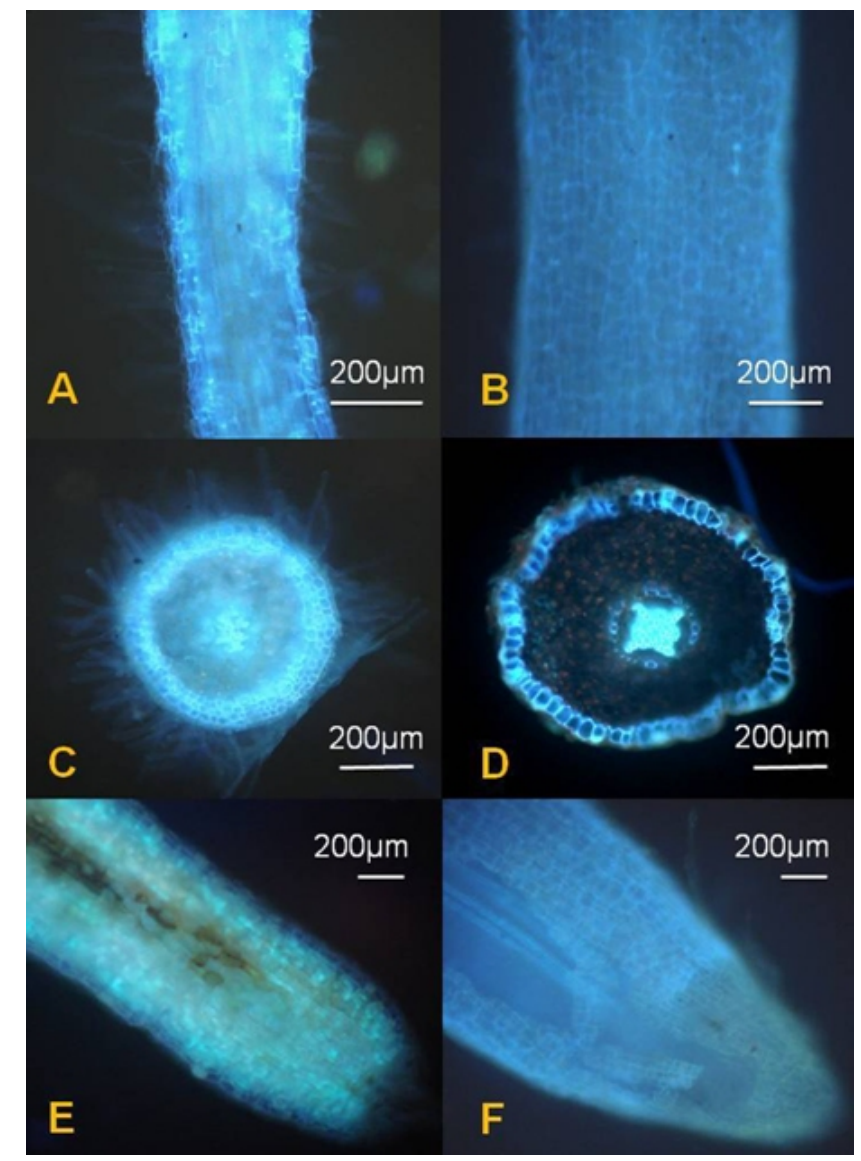

Figure 1. Localization of $\mathrm{CPT}$ in $\mathbf{A}, \mathbf{C}, \mathbf{E}$ : in vivo root; $\mathbf{B}, \mathbf{D}, \mathbf{F}$ : in vitro root

\subsection{Stem}

Stem showed accumulation of CPT in single layered epidermal as well as in the xylem region. Primary as well as secondary cortex does not show presence of prominent fluorescence. Stem also showed the presence of trichome with a prominent fluorescence suggesting presence of CPT. Trichomes were dense in in vivo sections where as in vitro sections showed negligible number of trichomes (Figure 2).

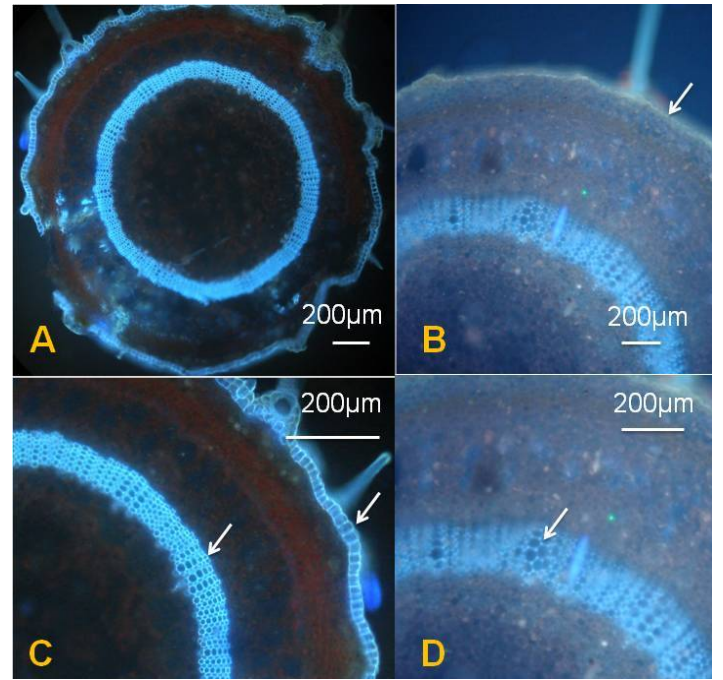

Figure 2. Localization of CPT in A, C: in vivo stem; B, D: in vitro stem

\subsection{Meristem}

Apical as well as axillary meristem showed prominent fluorescence at the tip region suggesting accumulation of CPT in both in vivo as well as in vitro sections (Figure 3A-F). The trichomes were prominently present and showed the presence of CPT in in vivo section; but in in vitro sections trichomes were absent unlike in the leaf, stem sections. The trichomes were uniserrate and multicellular (Figure 3F)

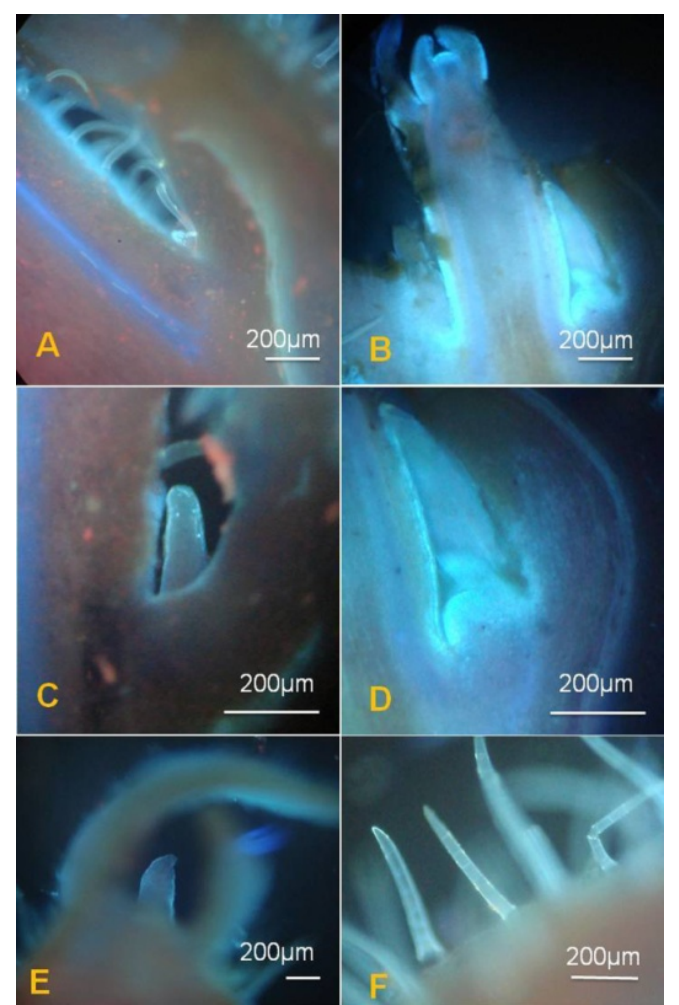

Figure 3. Localization of CPT in A, C: in vivo auxilary meristem, $\mathbf{E}$ : in vivo apical meristem; B, D: in vitro apical and auxiliary meristem, $\mathbf{F}$ : trichomes 


\subsection{Leaf}

In vivo leaves were covered with dense layer of trichome on abaxial and adaxial surface of leaves as well as petiole (Figures 4A, 5A). In vitro leaves and petiole showed less or no trichome on its surface (Figure 4B, 5B). In case of leaves we also could see that the mature sections show abundance of CPT content compared to young tissue (Figure 4C). Epidermal layer showed presence of CPT in both in vivo and in vitro sections. In the petiole sections we could observe that the xylem tissue showed characteristic blue colour suggesting the presence of CPT (Figure 5D).

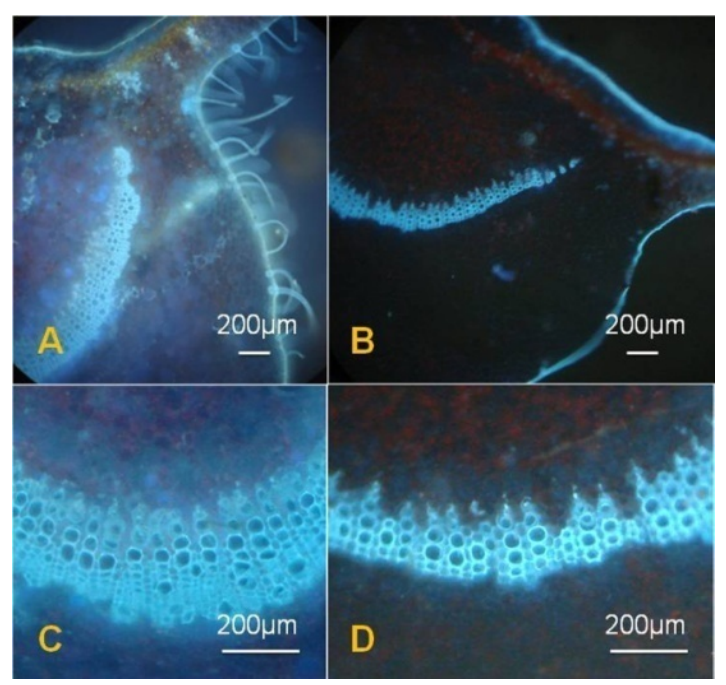

Figure 4. A, C: in vivo midrib; $\mathbf{B}, \mathbf{D}$, : in vitro midrib

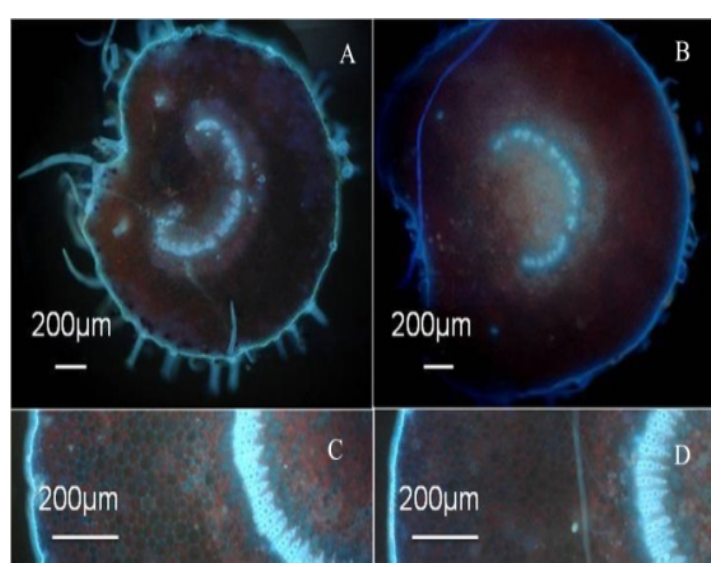

Figure 5. A, C: in vivo Petiole; B, D,: in vitro Petiole

\subsection{Quantification of CPT}

Quantification of CPT using HPLC and HPTLC (Figure 6-9) shows that the highest amount of CPT is present in root methanolic extract $\left(0.026 \% \pm 0.0005^{\mathrm{A}}\right.$ of dry weight $)$ followed by bark methanolic extract $\left(0.014 \% \pm 0.00043^{\mathrm{A}}\right)$. Where as in vitro cultures shoots shows $0.002 \% \pm 0.00004^{\mathrm{A}}$ of its dry weight and callus shows $0.001 \% \pm 0.00062^{\mathrm{A}}$ of its dry weight. Hexane extracts showed no or negligible presence of CPT for all the plant parts.

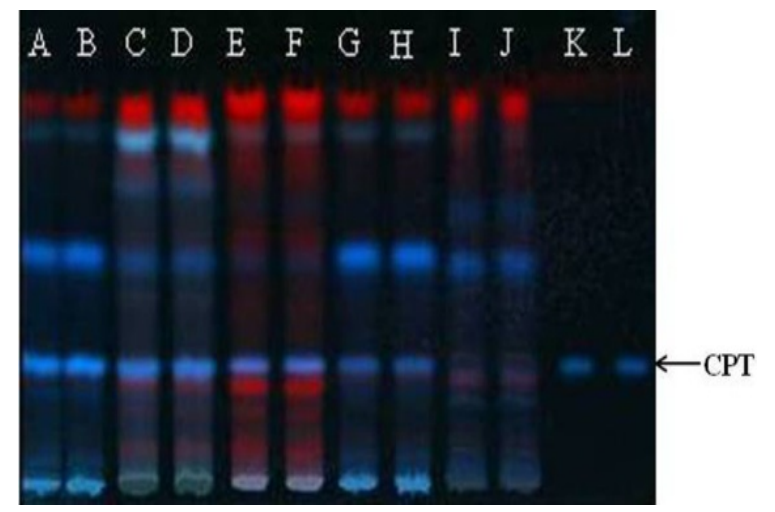

Figure 6. HPTLC of Methanolic extracts A,B: Methanolic roots; C,D: Methanolic stem bark; E,F: Methanolic leaves; G,H: Methanolic shoots; I,J: Methanolic callus; K,L: Camptothecin.

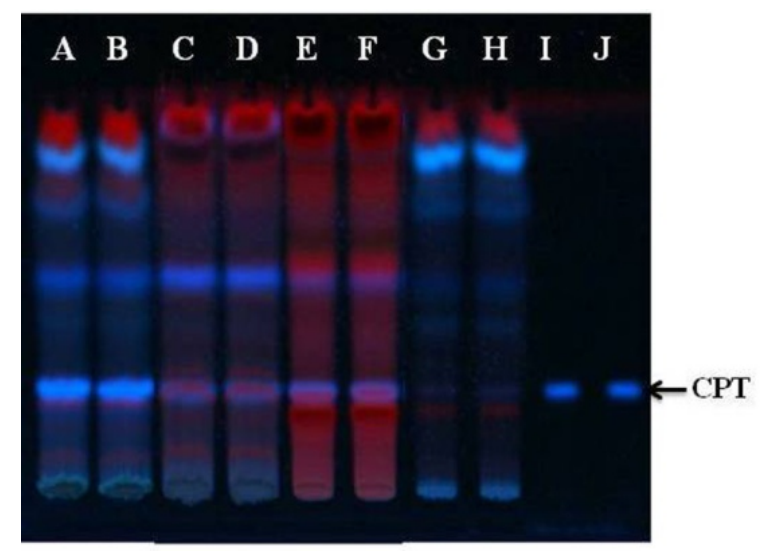

Figure 7. HPTLC of Stem bark extracts A,B: Methanolic crude; C,D: Ethyl acetate; E,F: Chloroform; G,H: Hexane; I,J: Camptothecin
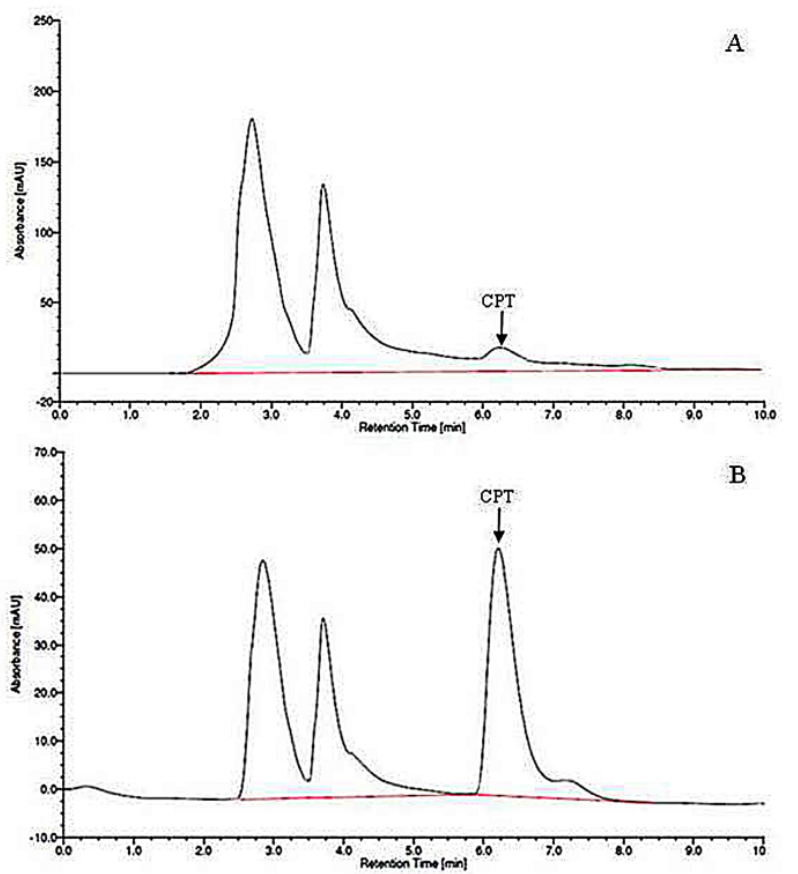

Figure 8. Representative HPLC chromatograms of extracts from A) Callus B) co-chromatogram of methanolic callus extract 


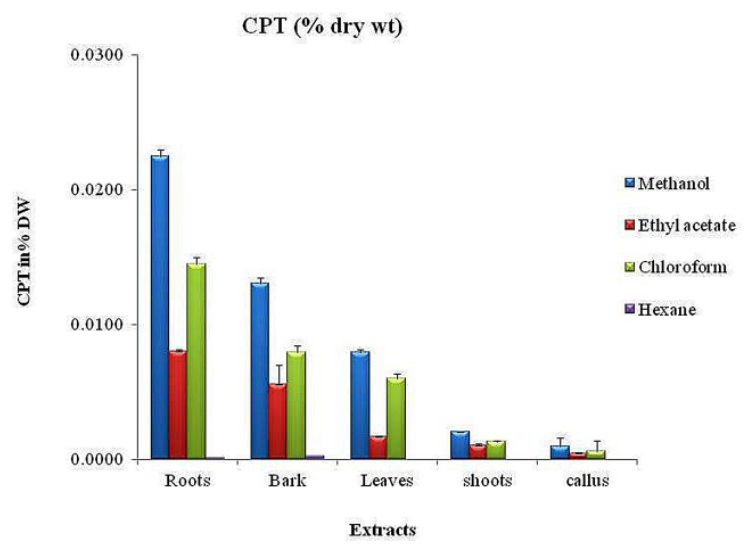

Figure 9. \% amount of CPT obtained from different plant parts of C. fragrans

\section{Discussion}

In recent years because of the heavy demand, there has been a serious threat of extinction of the populations of the trees producing CPT in the Western Ghats $\left(8^{\circ} \mathrm{N}\right.$ to $21^{\circ} \mathrm{N}$ latitudes) forests of south India [5]. Also increase in the clinical application of CPT and its analogues have increased the need to develop methods for higher production. As biosynthesis and accumulation of alkaloids are tissue-specific and cell type-specific [6,7] studies revealing the site of accumulation in a plant provide knowledge about the highest accumulating organ which could be used for further studies. In case of $C$. fragrans the young plant material showed less fluorescence whereas mature tissue showed more fluorescence in large number of cells, suggesting that maturity contributes towards high accumulation of CPT. So mature tissue was further considered for extraction of CPT. Presence of CPT in in vitro cultures indicates that in vitro systems can be used for further experiments so as to reduce overexploitation of natural population. Localization study has led to the conclusion that the root and stem bark portions are more useful to extract CPT. Quantification of CPT in this plant was comparable with the levels in current CPT sources in use. In all the organs studied, roots accumulated highest levels of CPT followed by stem bark. Tissue-wise, CPT tends to accumulate more in the root bark than root wood, possibly due to the high activities of an enzyme tryptophan synthase, responsible for the key intermediate tryptophan in the CPT biosynthesis pathways $[8,9]$ HPLC and HPTLC data showed that $C$. fragrans has significant level of CPT production in both in vivo as well as in vitro plant parts, manipulations of which could give enhanced metabolite production.

\section{Conclusion}

In C. fragrans CPT is accumulated in all the plant parts, especially in trichomes, epidermal region and vascular elements. Maturity contributes towards the accumulation of
CPT. Quantification studies showed that in all the plant parts studied roots accumulates highest amount of CPT followed by stem bark. In vitro shoots also showed presence of CPT which is considered as important finding as in vitro systems can further be used for manipulation studies like elicitation, precursor feeding and transformation to increase $\mathrm{CPT}$ production in in vitro cultures. This is the first report of localization of CPT, and quantification in serial extracts of in vivo and in vitro plant parts in C. fragrans giving basic information regarding extractability and site of accumulation of CPT.

\section{Acknowledgements}

The authors would like to acknowledge University Grant Commission, New Delhi for financial assistance.

\section{REFERENCES}

[1] A. V. Kulkarni, N. P. Malpathak. Micropropagation of Chonemorpha fragrans (Moon) Alston: An endangered medicinal plant, Indian Drugs, Vol.42, 1001-1003, 2006.

[2] E. Kjeldsen, J. Q. Svejstrup, Gromova II, J. Alsner, O. Westergaard. Camptothecin inhibits both the cleavage and relegation reactions of eukaryotic DNA topoisomerase, J Mol Biol, Vol.228, 1025-1030, 1992.

[3] E. Priel, S. D. Showalter, D. G. Blair. Inhibition of human immunodeficiency virus (HIV-1) replication in vitro by noncytotoxic doses of camptothecin, a topoisomerase I inhibitor, AIDS Res Hum Retroviruses, Vol.7, 65-72, 1991.

[4] A. V. Kulkarni, A. A Patwardhan, U. Lele, N. P. Malpathak. Production of camptothecin in cultures of Chonemorpha grandiflora, Pharmacognosy Res, Vol.2, 296-299, 2010.

[5] B. T. Ramesha, T. Amna, G. Ravikanth, R. P. Gunaga, R. Vasudeva, K. N. Ganeshaiah, R. Umashaanker, R. K. Khajuria, S. C. Puri, G. N. Qazi. Prospecting for Camptothecines from Nothapodytes nimmoniana in the Western Ghats, South India: identification of high-yielding sources of camptothecin and new families of camptothecines, J Chromatogr Sci, Vol.46, 362-368, 2008.

[6] V. De Luca, B. St Pierre. The cell and developmental biology of alkaloid biosynthesis, Trends Plant Sci, April 5, 168-173, 2000 .

[7] N. Samanani, C.Y. Edry weightard, J.F. Peter. Cell type-specific protoberberine alkaloid accumulation in Thalictrum flavum, J Plant Physiol, Vol.159, 1189-1196, 2002.

[8] Z. Liu, S. B. Carpenter, W. J. Bourgeois, Y. Yu, R. J. Constantin, M. J. Falcon, J. C. Adams. Variations in the secondary metabolite camptothecin in relation to tissue age and season in Camptotheca acuminate, Tree Physiol, Vol.18, 265-270, 1998.

[9] H. Lu, T. D. McKnight. Tissue-specific expression of the $\beta$-subunit of tryptophan synthase in Camptotheca acuminata, an indole alkaloid producing plant, Plant Physiol, Vol.120, 43-52, 1999. 